\title{
A rare entity of chronic ulcerative stomatitis - A case report
}

\author{
Vammi Sirisha \\ MDS (Oral Medicine and Radiology), Private Practitioner, Bangalore, Karnataka, India \\ *Corresponding Author: Vammi Sirisha \\ Email: luckycherry.siri@gmail.com
}

\begin{abstract}
Chronic ulcerative stomatitis (CUS) is an immune-mediated mucocutaneous disorder characterized clinically by erosions or ulcers involving mucosal surfaces and occasionally, the skin affecting 5\% of the population. About $14 \%$ of cases exhibits concurrent cutaneous lichen planus and biopsy proven and distinct immunofluorescent features. Microscopically, the lesions usually described as clinical entity of Erosive lichen planus, which is the second most common form of oral lichen planus followed after reticular type. This case report reviews the clinical approach of Erosive oral lichen planus and timely treatment continued. Patient was counselled about the risk of malignant transformation and immediate biopsy was performed and histopathologically diagnosed as chronic ulcerative stomatitis (CUS), which may be a variant of lichen planus or a distant disease.
\end{abstract}

Keywords: Chronic Ulcerative Stomatitis (CUS), Direct Immunofluorescent (DI), Stratified Epitheial Specific Antinuclear Antibody (SESANA), Hydroxychloroquine.

\section{Introduction}

Chronic ulcerative stomatitis (CUS) is a rare mucocutaneous disorder, usually associated with a painful oral mucosal ulcers or erosions. Most of the cases exhibit concurrent lesions involving the genital mucosa and conjunctiva; $5 \%$ of cases affects skin as cutaneous lichen planus and having distinct immunofluorescent features. ${ }^{1}$ chronic ulcerative stomatitis was first described by Jaren $\mathrm{KO}$ et $\mathrm{al}^{2}$ and exhibited clinically as a oral ulcerations that are often bordered by white striations as similar to erosive lichen planus. CUS can be distinguished from the other immune mediated vesiculobullous conditions by its very characteristic direct immunofluorescence pattern. $^{3}$

The distinction of CUS from other immune mediated condition is necessary, as CUS is generally not responsive to glucocorticosteroids, instead reacts favourably to hydroxychloroquine. ${ }^{4}$ The occurrence of CUS is usually between $6^{\text {th }}$ and $7^{\text {th }}$ decade of life, most commonly affecting females than males and generally persists as a chronic course with episodes of remission and exacerbation. ${ }^{5,6}$

The etiopathogenesis of CUS is not well defined, but may be due to auto-antibody reaction, presumably against a 70KDa epithelial nuclear protein, an isoform of p63 protein, plays an important role in apoptotic epithelial injury through the p53 pathway, which shows significant of its occurrence in the oral mucosa due to high epithelial turnover rate and risk for minor trauma. ${ }^{7,8}$

Any part of the oral mucosa may be affected, most frequently the tongue, the gingiva and the buccal mucosa. When affecting the gingiva, the appearence is often described as desquamative gingivitis. These oral lesions are usually widespread and are painful and associated with systemic symptoms such as weight loss, malaise, depression and apathy. ${ }^{9}$

Hence, it is important for all clinicians to be aware of the clinical presentations and provide prompt palliation to the chronic ulcerative stomatitis affecting the oral cavity. This article presents a long-standing case of an erosive form of oral lichen planus with desquamative gingivitis affecting a middle-aged female and she has been histopathologically diagnosed as chronic ulcerative stomatitis.

\section{Case Report}

A 50 year-old female patient had visited with a chief complaint of burning sensation of the entire oral cavity that started almost 1year back which was insidious in onset and moderate in nature and aggravated on having spicy food. Patient had a dental history of teeth sensitivity of her lower right and left back teeth since 2 years.

The patient had no relevant medical history. Personal history revealed that she had a mixed diet and had no deleterious habits. Patient gives a history of disturbed sleep since 2 years, as she was under considerable stress due to some family related issues.

Extra orally, there was no grossly changes or abnormalities detected (Fig. 1). On intra oral examination, presence of discrete raised papular lesions seen on the right and left side of the upper and lower vestibular regions and the dorsal surface of the tongue. Lesions are predominantly erythematous areas with peripherally bounded by straight sharp angular abruptly ending whitish striae (Fig. 2). Lesions also involving the marginal gingiva of all teeth on the buccal aspect and the lingual aspect of 31,32,41,42 regions. (Fig. 3).

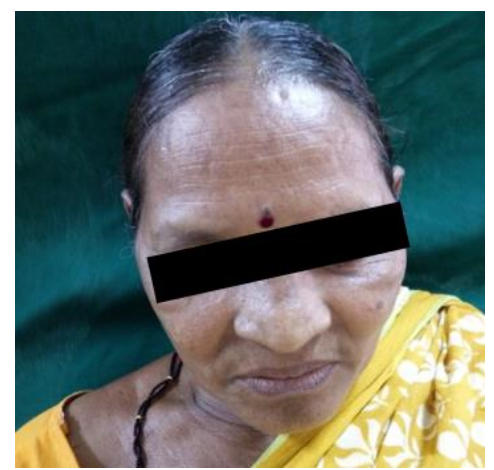

Fig. 1: Front profile view 

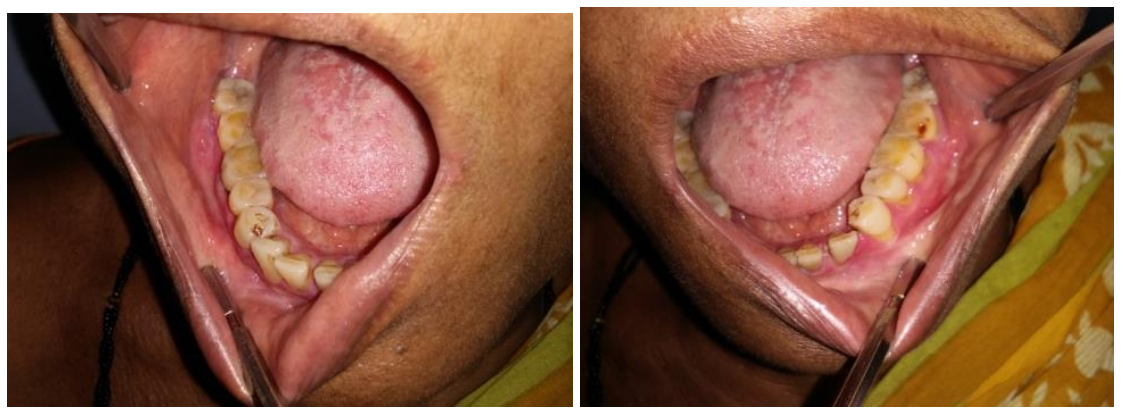

Fig. 2: Raised papules with erythematous areas peripherally bounded by whitish striations in angular pattern involving the right and left buccal vestibular regions and dorsal surface of the tongue.
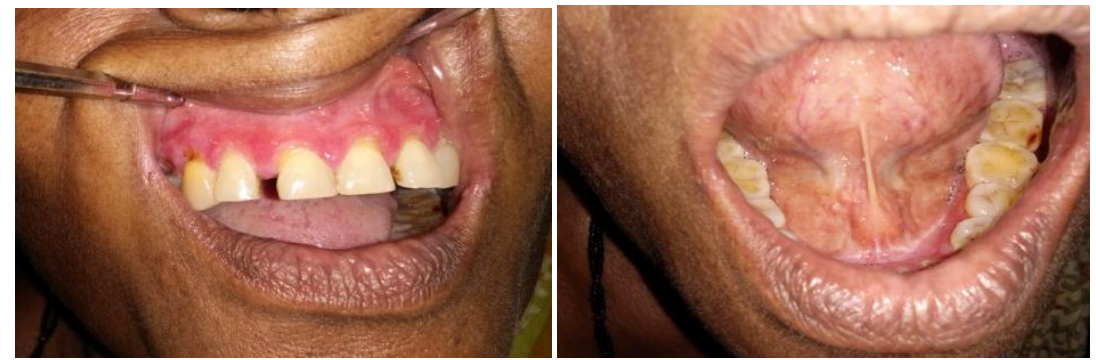

Fig. 3: Lesions also involving the marginal gingiva of all teeth on the buccal aspect and the lingual aspect of $31,32,41,42$ regions.

All the Inspectory findings were confirmed on palpation during which lesions were found to be non-scrapable and also mildly tender. Considering the clinical presentation and other related clinical history findings, the case was provisionally diagnosed as erosive lichen planus with desquamative gingivitis.

As Erosive type of oral lichen planus have a high risk of malignant transformation. The patient was convinced to undergo incisional biopsy. Before which all routine blood investigations were done. Fasting blood sugar and the blood pressure were found to be within the normal limits. Subsequent histopathological examination reveals that H\&E stained soft tissue sections shows ulcerated surface epithelium. The associated connective tissue contains numerous dense mixed inflammatory infiltrate predominantly lymphocytes. The connective tissue also exhibits blood capillaries and muscle fibres. All these features were consistent with that of CUS, thereby confirming our final diagnosis as CUS.

The patient early put on a course of topical corticosteroid therapy of $0.1 \%$ triamcinolone acetonide oral paste for initially 3 months. As no symptom relief, then the systemic administration of wysolone $40 \mathrm{mg}$ twice daily for 15 days was given, but not subsided completely. Later diagnosed as CUS, the therapeutic dose of $200 \mathrm{mg}$ of hydroxychloroquine per day was given for 10 days. Topical corticosteroids were added to the treatment regime, effectively lowering the dose of hydroxychloroquine required and therefore limiting possible side effects. During the recall visit, there was considerable remission of the lesion and the symptoms as well. She was educated about the chronicity and concurrent appearences of the lesions which warrants for the need for regular follow-up and was counselled for stress management.

\section{Discussion}

Chronic Ulcerative Stomatitis is typically a disease of middle-aged women (average age at diagnosis of 58.9 years) usually characterized by the presence of atrophic or erosive lesions of the oral mucosa that heal without scarring and display alternated periods of exacerbation and remission. ${ }^{10}$ The etiopathogenesis of CUS is not defined and it may probably due to which antinuclear antibodies are directed against the chronic ulcerative stomatitis protein (CUSP $\triangle \mathrm{Np} 63 \alpha$ ). As CUSP is an anti-apoptotic protein, inhibition of the action of CUSP by auto-antibody binding may lead to apoptotic epithelial injury through the p53 pathway. ${ }^{8}$

Oral lesions of CUS are characterised by erythematous macules, large tender erosions, ulcers and vesicles. Lesions may occur on keratinised and on non-keratinised mucosa commonly associated with pain. Patients may also suffer from weight loss, insomnia, malaise, depression, apathy, a feeling of helplessness, nervousness, fatigue and discomfort on eating and drinking. ${ }^{4}$ Lesions generally heal without scarring. 5

CUS may be indistinguishable clinically from oral LP, lichenoid stomatitis, mucous membrane pemphigoid, dermatitis herpetiformis, linear IgA disease, pemphigus vulgaris, erythema multiforme, Pyostomatitis vegetans, and epidermolysis bullosa acquisita. ${ }^{11}$

The light microscopic features (H\&E) of CUS are reported to be reminiscent and in some cases, indistinguishable from oral LP. The histopathological variability of CUS and oral LP warrants the use of DIF. ${ }^{12}$ Both circulating and tissue-bound IgG antibodies to a keratinocyte nuclear antigen are required for the diagnosis of CUS. ${ }^{10}$ 
With DIF, IgG antibodies in CUS are found bound to nuclei of keratinocytes of the basal and suprabasal epithelial cell layers. This IgG staining shows a unique stratified epithelial specificantinuclear antibody (SES-ANA) pattern. The SES-ANA pattern has a speckled or finely granular appearance usually in a perinuclear distribution. ${ }^{10}$

Chorzelski, one of the first authors to describe CUS in 1990, proposed major and minor diagnostic criteria to facilitate diagnosis. The major criterion for diagnosis of CUS is the presence of erosive oral lesions with the characteristic SES-ANA pattern on using DIF. ${ }^{13}$ A good response to treatment with hydroxychloroquine or hydroxychloroquine (chloroquine) combined with corticosteroid. ELISA may become the diagnostic test of choice in future since a recent experimental study has demonstrated excellent sensitivity and specificity. ${ }^{14}$

The use of glucocorticoids in the treatment of CUS may not be satisfactory, but may provide some symptomatic relief and clinical improvement. The treatment of choice is hydroxychloroquine which may bring about remission in up to $70 \%$ of patients.

Therapeutic doses of 200mg hydroxychloroquine (or chloroquine if hydroxychloroquine is unavailable) per day are recommended. ${ }^{10}$

The main action of the hydroxyl chloroquine is to reduced the antigen presentation down-regulates the immune response leading to improvement of the immune-mediated lesions and may be associated with the side effects of the irreversible retinopathy, toxic psychosis, neuromyopathy, agranulocytosis and aplastic anaemia. ${ }^{15}$

\section{Conclusion}

The evidence to support the argument that chronic ulcerative stomatitis is not a distinct entity, but rather a variant of lichen planus, because the oral lichen planus and chronic ulcerative stomatitis both clinically and histopathologically. As both the lesions are concurrently affected by the same immunopathogenic reaction directed at the same nuclear selfantigen of epithelial cells, giving rise to lesions due to the circulating antibodies to the $\Delta \mathrm{Np} 63 \alpha$ protein. Furthermore, the apparent variant of lichen planus currently termed chronic ulcerative stomatitis, there is an additional immunopathogenic mechanism involved, namely the $\Delta \mathrm{Np} 63 \alpha$-specific IgG autoimmune reaction.

\section{Clinical significance}

As chronic ulcerative stomatitis is most frequently encountered as Erosive lichen planus. The rarity of the variants and their atypical presentations make their timely diagnosis and management more difficult in the clinical setting. Oral lesions are chronic, rarely associated with immune mediated vesiculo-bullous lesions, which makes the condition difficult to palliate and may persists for several years.

This case report reviews the clinical approach of CUS and how to differentiate from oral lichen planus, wherein, the patient was educated, counselled about the chronicity of the condition and timely management of the condition.

\section{Source of funding}

None.

\section{Conflict of interest}

None.

\section{References}

1. Carlson MW, Garlick JA, Solomon LW. Chronic ulcerative stomatitis: evidence of autoimmune pathogenesis. Oral Surg Oral Med Oral Pathol Oral Radiol Endod 2011;111:742-8.

2. Jaremko WM, Beutner EH, Kumar V, Kipping H, Condry P, Zeid MY, et al. Chronic ulcerative stomatitis associated with a specific immunologic marker. J Am Acad Dermatol 1990;22: 215-20.

3. Solomon LW. Chronic ulcerative stomatitis. Oral Dis 2008;14:383-9.

4. Islam MN, Cohen DM, Ojha J, et al. Chronic ulcerative stomatitis: diagnostic and management challenges--four new cases and review of literature. Oral Surg Oral Med Oral Pathol Oral Radiol Endod 2007;104:194-203.

5. Solomon LW, Aguirre A, Neiders M, et al. Chronic ulcerative stomatitis: clinical, histopathologic, and immunopathologic findings. Oral Surg Oral Med Oral Pathol Oral Radiol Endod 2003;96:718-26.

6. Parodi A, Cozzani E, Massone C. Prevalence of stratified epithelium-specific antinuclear antibodies in 138 patients with lichen planus. J Am Acad Dermatol 2007;56:974-8.

7. Lee LA, Walsh P, Prater CA, Su LJ, Marchbank A, Egbert TB, et al. Characterization of an autoantigen associated with chronic ulcerative stomatitis: the CUSP autoantigen is a member of the p53 family. J Invest Dermatol 1999;113:14651.

8. Solomon LW, Neiders ME, Zwick MG, et al. Autoimmunity to deltaNp63alpha in chronic ulcerative stomatitis. J Dent Res 2007;86:826-31.

9. Fourie J, van Heerden WF, McEachen SC, et al. Chronic ulcerative stomatitis: a distinct clinical entity? SADJ 2011;66:119-21.

10. Chorzelski TP, Olszewska M, Jarzabek-Chorzelska M, Jablonska S. Is chronic ulcerative stomatitis an entity? Clinical and immunological findings in 18 cases. Eur J Dermatol 1998;8: 261-5.

11. Scully C, Porter SR. The clinical spectrum of desquamative gingivitis. Semin Cutan Med Surg 1997;16:308-13.

12. Scully C, Beyli M, Ferreiro MC, Ficarra G, Gill Y, Griffiths M, et al. Update on oral lichen planus: etiopathogenesis and management. Crit Rev Oral Biol Med 1998;9:86-122.

13. Chorzelski TP. Chronic ulcerative stomatitis (CUS): a new disease entity with specific immunological marker (SESANA). Przegl Dermatol 1990;77:229-32.

14. Solomon LW, Stark PC, Winter L. ELISA test for p63 antibodies in chronic ulcerative stomatitis. Oral Dis 2010;16:151-5.

15. Fox RI. Mechanism of action of hydroxychloroquine as an antirheumatic drug. Semin Arthritis Rheum 1993;23:82-91.

\footnotetext{
How to cite this article: Sirisha V. A rare entity of chronic ulcerative stomatitis - A case report. Int J Oral Health Dent $2019 ; 5(4): 233-5$.
} 